\section{Is Progressive Multiple Sclerosis a Gray Matter Disease?}

Over the past few years, it has become painfully clear that there is much more to the multiple sclerosis (MS) brain than meets the eye on conventional magnetic resonance (MR) images. Apart from the typical focal lesions in the periventricular white matter (WM), substantial abnormalities can also be found in cortical and subcortical gray matter (GM), and in the "normalappearing” WM (NAWM). ${ }^{1,2}$ Histopathological studies have shown that cortical demyelination may be extensive in chronic MS cases, and in some cases, a pattern of general cortical demyelination may develop, which means that nearly all cortical gyri are affected. ${ }^{3}$ Demyelinated cortical lesions may be mixed, that is, they involve both (lower layers of) the cortex and areas of subcortical WM (so-called type I lesions), but more often they are purely intracortical (lesion types II, III, and IV). ${ }^{1}$ In a recent postmortem study, it was shown that in the progressive phase of the disease, type III subpial cortical demyelination becomes more prominent and cooccurs with a pattern of diffuse microglial reactivity throughout the WM. ${ }^{2}$ Clinically, GM demyelination is of relevance, because it was found that the WM abnormalities visible on MRI cannot explain the full extent of clinical, including cognitive, deficits in MS patients. ${ }^{4}$

It is difficult to detect cortical GM lesions on conventional T2-weighted or even on more advanced fluid-attenuated inversion recovery images because these lesions are largely noninflammatory and generate only little contrast on MRI. ${ }^{5}$ However, recent studies have shown that an improvement in cortical lesion detection in MS can be achieved by using a double inversion recovery ${ }^{6,7}$ or T1-weighted, three-dimensional, spoiled gradient recalled echo sequence. ${ }^{8,9}$ Apart from visualizing cortical lesions, GM damage can be assessed by using quantitative magnetic resonance imaging (MRI) techniques ${ }^{10-12}$ or atrophy and cortical thickness measurements, ${ }^{13,14}$ which generally correlate well with disability. ${ }^{10,15}$ Cortical thickness studies have shown focal thinning of frontotemporal, parietal, precentral, and anterior cingulate cortex, ${ }^{14,16}$ largely consistent with histopathological data on the preferential distribution of cortical demyelination in MS. ${ }^{3}$ Brain atrophy was reported to be already present in patients with a clinically isolated syndrome (CIS) ${ }^{17}$ and to further accrue with disease progression. ${ }^{18,19}$ These results notwithstanding, several issues regarding the (separate) evolution of WM and GM atrophy among different MS disease types and stages, the relation between GM and WM atrophy, and their respective effects on disability are still largely unclear.

In this issue of the Annals of Neurology, two interesting articles deal with these issues. ${ }^{20,21}$ In Fisniku and colleagues $^{20}$ study, a cohort of MS patients with a uniquely long follow-up duration was evaluated to assess tissue-specific atrophy. The patients from this cohort initially presented with a clinically isolated syndrome (CIS) and were subsequently followed for 20 years, undergoing clinical and MRI evaluations at approximately 5 -year intervals. The investigators found that the extent of GM atrophy in the MS patients was greater than that of WM atrophy after 20 years of disease, and that there was significantly more GM atrophy, but not WM atrophy, in secondary progressive MS (SPMS) versus relapsing remitting MS (RRMS) patients, as well as in RRMS versus CIS patients who had not had a second episode. The clinical relevance of these findings is illustrated by significant correlations between GM, but not WM, atrophy and disability as measured by Expanded Disability Status Scale and Multiple Sclerosis Functional Composite. GM atrophy proved to be a stronger predictor of disability than focal WM lesion load and WM atrophy. These findings emphasize the need for a better understanding of (increasing) GM damage in MS, as well as the potential usefulness of GM atrophy measurements in natural history studies and treatment trials.

Fisniku and colleagues' ${ }^{20}$ study demonstrates that, relative to the WM, GM atrophy is more abundant and more clinically significant in progressive compared with relapsing disease. However, because of its crosssectional design, this study could not provide any information on the rate of GM atrophy. Relatively little is known about GM atrophy rates in MS, although several studies have investigated rates of whole-brain atrophy, ${ }^{15,22,23}$ which appears to predict future clinical status. ${ }^{15,24}$ In Fisher and coworkers ${ }^{21}$ study, also featured in this issue of the Annals, GM atrophy was studied longitudinally in a large group of MS patients who were followed over 4 years, and the authors distinguish between GM and WM atrophy rates across the main MS disease types. They found that, although WM atrophy rate remained constant at threefold normal across all disease stages in their dataset, GM atrophy rate accelerated in the SP phase. Compared with control subjects, a 3.4-fold increase of GM atrophy rate was seen in CIS patients converting to RRMS, whereas a 14-fold increase was measured in SPMS patients. These results, showing that the character of the pathological process changes with advancing disease, with a dominance of GM pathology in the progressive phase, complement Fisniku and colleagues ${ }^{20}$ findings. Interestingly, Fisher and coworkers ${ }^{21}$ showed that WM 


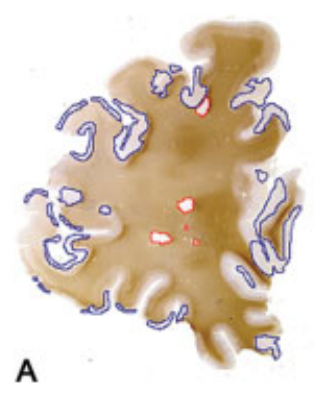

A

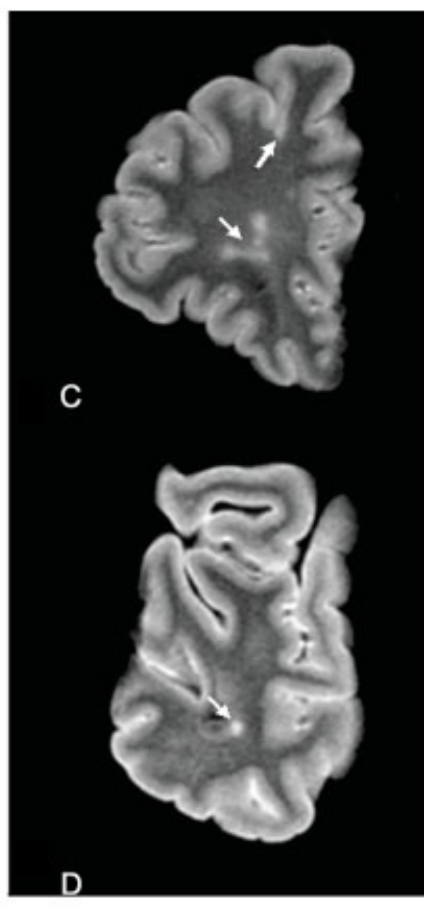

B

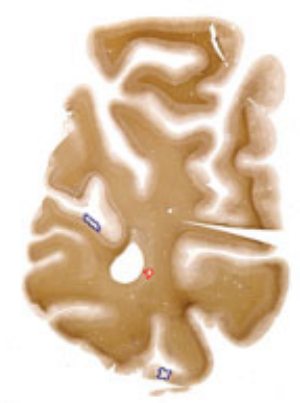

Fig. Paraffin sections immunohistochemically stained with anti-proteolipid protein antibody $(A, C)$ and corresponding T2-weighted magnetic resonance $(M R)$ images from a patient with chronic extensive cortical subpial demyelination $(A, B)$ and from a patient with little cortical demyelination $(C, D)$. Demyelinated cortical areas are visible in immunohistochemical stainings ( $A, C$; blue outlines) but not on $M R$ imaging $(B, D)$. White matter demyelination ( $A, C$; red outlines), detectable by MR imaging ( $B, D$; arrows), did not correlate with the extent of cortical gray matter demyelination. (Reprinted from Bo and colleagues, ${ }^{25}$ by permission.)

lesion volume change, WM lesion magnetization transfer ratio, and magnetization transfer ratio of the normal-appearing brain tissue together accounted for a large proportion of the variance in whole-brain and WM atrophy in RRMS and SPMS. In RRMS, these same parameters explained part of the GM atrophy as well. However, no WM correlates were observed for GM atrophy in SPMS patients. All this appears to indicate that GM and WM disease processes are largely unrelated in progressive MS, which confirms histopathology findings where extensive cortical demyelination was found to be unrelated to WM lesion load (Fig). ${ }^{25}$

Fisher and coworkers ${ }^{21}$ hypothesize that lesions intrinsic to the GM, not visible on their standard MR sequences, are the major contributors to GM atrophy in progressive MS, and suggest that more advanced MRI techniques be applied in future studies to investigate the relation between GM lesions and GM atrophy. Although such a relation may likely exist, and this issue certainly warrants further investigation, there have also been reports of pathology outside of focal GM le- sions. ${ }^{26,27}$ For example, in a recent histopathology study, Wegner and coauthors ${ }^{27}$ describe a $10 \%$ overall cortical thickness reduction in their MS cases, which was, at least partly, independent of cortical demyelination. Substantial atrophy with neuronal loss and neuronal shape changes were also found in the MS hippocampus, ${ }^{28}$ a nonneocortical structure that is frequently and extensively demyelinated in $\mathrm{MS}^{29}$ These changes were more prominent within hippocampal lesions but were also present in nondemyelinated areas. A marked reduction of synapses, suggestive of deafferentation, was found in both demyelinated and normally myelinated MS hippocampus. Furthermore, on a more methodological note, the diffuse and subtle WM inflammation that accompanies increasing GM demyelination in the progressive phase ${ }^{2}$ may remain largely undetected by measures such as magnetization transfer ratio. ${ }^{30}$ It should be further investigated whether other, perhaps more sensitive, quantitative MR techniques such as T1-relaxometry or diffusion tensor imaging are better capable of detecting these abnormalities in the WM, and whether they are related to the disproportionate increase of GM atrophy in progressive MS.

With these results in hands, it is now important to explore the relative contributions of GM demyelination, neuronal shrinkage/loss, and deafferentation to overall GM atrophy and cortical thinning in MS. Also, the effect of subtle, diffuse WM abnormalities on GM atrophy remain to be addressed in future studies. Meanwhile, Fisniku and colleagues ${ }^{20}$ and Fisher and coworkers' $^{21}$ studies have importantly advanced GM research in MS. By using MRI to show that GM atrophy accumulates and even accelerates with disease progression, independently of WM disease, and that GM atrophy is more strongly predictive of clinical disability than WM abnormalities, the investigators have finalized a conclusion that was already hinted at but could not be definitively proved by histopathology.

I always assumed that histology is the gold standard; this is the world turned upside down.

Jeroen J. G. Geurts, PhD

Departments of Pathology and Radiology

VU University Medical Center

Amsterdam, The Netherlands

\section{References}

1. Bo L, Vedeler CA, Nyland H, et al. Intracortical multiple sclerosis lesions are not associated with increased lymphocyte infiltration. Mult Scler 2003;9:323-331.

2. Kutzelnigg A, Lucchinetti CF, Stadelmann C, et al. Cortical demyelination and diffuse white matter injury in multiple sclerosis. Brain 2005;128:2705-2712.

3. Bo L, Vedeler CA, Nyland HI, et al. Subpial demyelination in the cerebral cortex of multiple sclerosis patients. J Neuropathol Exp Neurol 2003;62:723-732. 
4. Barkhof F. The clinico-radiological paradox in multiple sclerosis revisited. Curr Opin Neurol 2002;15:239-245.

5. Geurts JJ, Bo L, Pouwels PJ, et al. Cortical lesions in multiple sclerosis: combined postmortem MR imaging and histopathology. AJNR Am J Neuroradiol 2005;26:572-577.

6. Geurts JJ, Pouwels PJ, Uitdehaag BM, et al. Intracortical lesions in multiple sclerosis: improved detection with $3 \mathrm{D}$ double inversion-recovery MR imaging. Radiology 2005;236:254-260.

7. Calabrese M, De Stefano N, Atzori M, et al. Detection of cortical inflammatory lesions by double inversion recovery magnetic resonance imaging in patients with multiple sclerosis. Arch Neurol 2007;64:1416-1422.

8. Bagnato F, Butman JA, Gupta S, et al. In vivo detection of cortical plaques by MR imaging in patients with multiple sclerosis. AJNR Am J Neuroradiol 2006;27:2161-2167.

9. Nelson F, Poonawalla AH, Hou P, et al. Improved identification of intracortical lesions in multiple sclerosis with phasesensitive inversion recovery in combination with fast double inversion recovery MR imaging. AJNR Am J Neuroradiol 2007; 28:1645-1649.

10. Vrenken H, Geurts JJ, Knol DL, et al. Whole-brain T1 mapping in multiple sclerosis: global changes of normal-appearing gray and white matter. Radiology 2006;240:811-820.

11. Agosta F, Rovaris M, Pagani E, et al. Magnetization transfer MRI metrics predict the accumulation of disability 8 years later in patients with multiple sclerosis. Brain 2006;129:2620-2627.

12. Rovaris M, Judica E, Gallo A, et al. Grey matter damage predicts the evolution of primary progressive multiple sclerosis at 5 years. Brain 2006;129:2628-2634.

13. De Stefano N, Matthews PM, Filippi M, et al. Evidence of early cortical atrophy in MS: relevance to white matter changes and disability. Neurology 2003;60:1157-1162.

14. Chen JT, Narayanan S, Collins DL, et al. Relating neocortical pathology to disability progression in multiple sclerosis using MRI. Neuroimage 2004;23:1168-1175.

15. Fisher E, Rudick RA, Simon JH, et al. Eight-year follow-up study of brain atrophy in patients with MS. Neurology 2002; 59:1412-1420.

16. Sailer M, Fischl B, Salat D, et al. Focal thinning of the cerebral cortex in multiple sclerosis. Brain 2003;126:1734-1744.

17. Anderson VM, Fernando KT, Davies GR, et al. Cerebral atrophy measurement in clinically isolated syndromes and relapsing remitting multiple sclerosis: a comparison of registration-based methods. J Neuroimaging 2007;17:61-68.

18. Rudick RA, Fisher E, Lee JC, et al. Use of the brain parenchymal fraction to measure whole brain atrophy in relapsingremitting MS. Multiple Sclerosis Collaborative Research Group. Neurology 1999;53:1698-1704.

19. Miller DH, Barkhof F, Frank JA, et al. Measurement of atrophy in multiple sclerosis: pathological basis, methodological aspects and clinical relevance. Brain 2002;125:1676-1695.

20. Fisniku LK, Chard DT, Jackson JS, et al. Grey matter atrophy is related to long term disability in multiple sclerosis. Ann Neurol 2008;64.

21. Fisher E, Lee J-C, Nakamura K, et al. Gray matter atrophy in multiple sclerosis: a longitudinal study. Ann Neurol 2008;64.

22. Horsfield MA, Rovaris M, Rocca MA, et al. Whole-brain atrophy in multiple sclerosis measured by two segmentation processes from various MRI sequences. J Neurol Sci 2003;216: 169-177.

23. Jasperse B, Minneboo A, de Groot V, et al. Determinants of cerebral atrophy rate at the time of diagnosis of multiple sclerosis. Arch Neurol 2007;64:190-194.

24. Minneboo A, Jasperse B, Barkhof F, et al. Predicting short-term disability progression in early multiple sclerosis: added value of MRI parameters. J Neurol Neurosurg Psychiatry 2008;79: 917-923.
25. Bo L, Geurts JJ, van der Valk P, et al. Lack of correlation between cortical demyelination and white matter pathologic changes in multiple sclerosis. Arch Neurol 2007;64:76-80.

26. Peterson JW, Bo L, Mork S, et al. Transected neurites, apoptotic neurons, and reduced inflammation in cortical multiple sclerosis lesions. Ann Neurol 2001;50:389-400.

27. Wegner C, Esiri MM, Chance SA, et al. Neocortical neuronal, synaptic, and glial loss in multiple sclerosis. Neurology 2006; 67:960-967.

28. Papadopoulos D, Dukes S, Patel R, et al. Substantial archaeocortical atrophy and neuronal loss in multiple sclerosis. Brain Pathol "Brain Pathol 2008, May 20. [E-Pub ahead of print]."

29. Geurts JJ, Bo L, Roosendaal SD, et al. Extensive hippocampal demyelination in multiple sclerosis. J Neuropathol Exp Neurol 2007;66:819-827.

30. Vrenken H, Geurts JJ, Knol DL, et al. Normal-appearing white matter changes vary with distance to lesions in multiple sclerosis. AJNR Am J Neuroradiol 2006;27:2005-2011.

DOI: 10.1002 /ana.21485

\section{Neonatal Encephalitis and White Matter Injury: More Than Just Inflammation?}

In this issue of Annals, Verboon-Maciolek and coworkers $^{1}$ show that human parechovirus $(\mathrm{HPeV})$, specifically $\mathrm{HPeV} 3$, is an important cause of neonatal viral encephalitis. The six serotypes of $\mathrm{HPeV}$ s that are included in the genus Parechovirus are small, singlestranded RNA (ssRNA) viruses belonging to the family Picornaviridae. ${ }^{2,3}$ These viruses bear many similarities with another and better known Picornaviridae genus, Enterovirus (EV). Indeed, the genus Parechovirus began with the reclassification of echoviruses 21 and 22 as $\mathrm{HPeV} 1$ and HPeV2 because of molecular and genetic differences from the remainder of EV. These differences are important because they explain, in part, why the usual polymerase chain reaction testing for EV does not detect $\mathrm{HPeV}$. Thus, encephalitic infection by $\mathrm{HPeV} 3$ has been overlooked in the past, which is one important point of Verboon-Maciolek and coworkers' ${ }^{1}$ article. (Neonatal encephalitis by other $\mathrm{HPeV}$ subtypes is extremely rare and is not discussed further.) The encephalitis caused by $\mathrm{HPeV} 3$ infection, as well as by $\mathrm{EV}$, is associated with neonatal seizures and with apparent cerebral white matter injury. ${ }^{1,4}$ VerboonMaciolek and coworkers ${ }^{1}$ report has important implications concerning the cause of neonatal viral encephalitis, the differential diagnosis of neonatal seizures, and the pathology and pathophysiology of the white matter injury. 